\title{
An Analysis of Perceived and Actual Anticoagulant Knowledge among Independent Pharmacy Patients
}

Kenric B. Ware, PharmD ${ }^{1}$; Marty Faile, PharmD²; Carrie Lynch, PharmD ${ }^{3}$

${ }^{1}$ South University School of Pharmacy, Columbia, SC; ${ }^{2}$ Sarasota Memorial Healthcare System, Sarasota, FL;

${ }^{3}$ Hawthorne Pharmacy, Columbia, SC

\begin{abstract}
Background: Deficits in knowledge of parameters surrounding anticoagulant therapy administration carry substantial risks for patient harm. Patients prescribed anticoagulants may overestimate their knowledge of anticoagulant use principles. The purpose of this study was to analyze independent pharmacy patients' perceived versus actual knowledge of their anticoagulation therapies. Methods: A 15-item survey across a dual-site pharmacy location evaluated patients' confidence in their understanding of anticoagulant prescribing parameters and their awareness of measures to take to safeguard anticoagulant therapy routines. $A$ pharmacist reviewed patient responses to survey items referencing actual understanding of anticoagulant therapies at the conclusion of the survey. Data analyses occurred by gender, age, ethnicity, location, living situation, self-management of anticoagulants, patient perception of anticoagulant safety, and reported receipt of anticoagulant education. Linear regressions were used for statistical analyses, with significance set at $p<.05$.

Results: During the span of three months, 45 patients, 20 females and 25 males, completed the survey. Perceived anticoagulant knowledge did not differ significantly by demographics. Actual anticoagulant knowledge declined by over $20 \%$ between age groups 60-69 and 80-89 years old. African Americans displayed 10\% lower actual anticoagulant knowledge than Caucasian Americans. Conclusions: This study revealed gaps in knowledge of anticoagulants among patients at an independent pharmacy. An appeal remains for more strategies to assist with knowledge of anticoagulant regimens. Healthcare institutions should continue to incorporate programs geared towards ongoing anticoagulant education, such as question and answer sessions, along with peer support mediums that foster optimal outcomes.
\end{abstract}

Keywords: Anticoagulant; survey; health literacy; adherence; monitoring

\section{Background}

Patients prescribed anticoagulants may not be too familiar with the safety and efficacy of their associated therapies. ${ }^{1-2}$ Despite this lack of understanding medications designed to manage coagulation-related conditions continue to enter the market. ${ }^{3-5}$ Opportunities therefore remain to increase knowledge of anticoagulants among patients within different healthcare systems such as pharmacies. ${ }^{6}$ It is beneficial for patients in pharmacies and other settings to have a better grasp of the details about anticoagulant regimens that often consist of side effects that may be experienced. ${ }^{7-8}$ It is also worthwhile for patients to be able to recognize anticoagulant-related concerns that warrant notifying healthcare personnel. ${ }^{9}$ Increased alertness to possible signs of adverse therapy events can foster greater adherence and empower patients to use their anticoagulant regimens with greater clarity and confidence. ${ }^{6}$

Strategies to address patients' missed doses, disease state comprehension, drug interactions, and side effect recognition have room for improvement. ${ }^{10-13}$ Literature reports indicate an inability to remember important points about anticoagulant

Corresponding author: Kenric B. Ware, PharmD

South University School of Pharmacy

Columbia, SC

Email: kbware@southuniversity.edu regimens can lead to suboptimal outcomes. ${ }^{14-15}$ Furthermore, recall of anticoagulant information for a brief period may overestimate patients' comfort level with their prescribed regimens. $^{6,16}$ In addition, diminished health literacy has the potential to affect patients' understanding of their anticoagulation care. ${ }^{12,15}$ Equally as important, disparities exist among patient comprehension levels of anticoagulants by racial demographics. ${ }^{17-18}$

Paradoxically, healthcare systems might exacerbate deficits in anticoagulant knowledge through inattention to clarifying questions coupled with staffing challenges and inadequate communication to illiterate patients. ${ }^{19}$

It is also valuable to understand why patients adhere to anticoagulant regimens to varying degrees aside from their lack of knowledge. Evidence suggests that perceived reductions in quality of life might result from cumbersome anticoagulation management. ${ }^{20}$ Therefore, limiting strict monitoring parameters and dietary considerations may enhance patients' abilities to incorporate anticoagulants into their daily routines. ${ }^{21}$ Conversely, routine monitoring of anticoagulants may equate to a reliable predictor of efficacy and safety. ${ }^{22}$ Patients also commonly experience similar challenges with taking their anticoagulants on a regular basis. ${ }^{23}$ Peer support models therefore could help to encourage anticoagulant adherence along with proper understanding and acceptance of 
established diagnoses. ${ }^{23}$ Moreover, patients receiving care from a limited number of medical providers in conjunction with having their anticoagulants filled at a single pharmacy institution have suffered fewer adverse effects likely due to better adherence. ${ }^{24}$ The purpose of this project was to assess differences in perceived and actual knowledge of patients filling anticoagulant medications within an independent pharmacy institution.

\section{Methodology}

This study took place at an independent pharmacy that operates across two locations, one in Camden, South Carolina and one in Lugoff, South Carolina. The patients completed a survey to determine their perceived understanding of their anticoagulants. The authors developed the survey based upon their independent and collective reviews of the medical literature. Communications to prospective patients inviting them to enroll in the study stemmed from medication refill histories of the following medications: warfarin, apixaban, or rivaroxaban. Two of the authors of this study generated a report from the electronic health information system at each pharmacy location of all prospective patients with refills of the aforementioned medications due in the upcoming two-month period. The same two authors contacted these prospective patients by telephone to explain the intent of the study and to assess their interest in being included. After patients verbally confirmed their willingness to participate, they received typewritten surveys attached to their medication packages designated for dispensing upon arrival.

The survey consisted of 15 items, divided into two categories: 5 items pertaining to perceived anticoagulant knowledge and 10 items relating to actual anticoagulant knowledge (Appendix A). A numbering system within the survey helped to identify patients for future data analysis during the study period. Pharmacists at each location received a copy of the survey to reference in future counseling sessions. The pharmacists prior to survey completion obtained verbal and written informed consent from all patients. A conversation between patients and the pharmacist-on-duty followed survey completions; clarifying anticoagulant prescribing details along with other medicationrelated concerns.

Percentage scores of patients' perceived anticoagulant knowledge resulted from calculating the sum of Likert scale items (maximum of five points each) and dividing the quantity by 25 then multiplying the resulting amount by 100 . Higher percentage scores indicated higher perceptions of anticoagulant knowledge. Percentage scores of patients' actual anticoagulant knowledge resulted from calculating the sum of total number of answers correct (maximum of one point each) and dividing this quantity by 10 then multiplying the resulting amount by 100 . Similarly, higher percentage scores indicated greater actual anticoagulant knowledge.
Demographic factors analyzed by perceived and actual anticoagulant knowledge included age, pharmacy location, gender, and ethnicity. Adherence factors analyzed by perceived and actual anticoagulant knowledge comprised living situation, self-management of anticoagulants, patient perception of anticoagulant safety, and reported education received about anticoagulant medications. A statistical comparison of both demographic and adherence factors with perceived and actual anticoagulant understanding was conducted using linear regressions. All data analyses were conducted using the Statistical Package for Social Scientists (SPSS), Version 24, with significance defined as a $p$-value of $<.05$. This study received institutional review board approval prior to implementation.

\section{Results}

Table 1 displays study demographics based in an independent pharmacy, across two locations, in South Carolina. With a roughly equal distribution between both locations, males outnumbered females. Patients 60 years old and above comprised approximately $80 \%$ of the study sample. Twice as many Caucasian American patients participated than African Americans. Almost three times as many patients lived with family members versus residing alone. No patients reported residence within an institution/facility. An excess of $90 \%$ of patients attested to managing their own medications. Less than one-fifth of patients did not feel safe taking their medications. Three times as many patients received anticoagulant education as opposed to those who did not.

Table 2 presents perceived knowledge of anticoagulants among patients. Average percent correctness did not apply to these survey responses. Self-perceptions of knowledge behaved as points of reference as opposed to accuracy and inaccuracy. Selfperceptions of anticoagulant knowledge was largely uniform among all study demographics $(p>.05)$. Males' higher perceptions of their anticoagulant knowledge than females came the closest to significant difference $(p=.09)$.

Table 3 features average actual knowledge, on a 100\% scale, exhibited by patients about their anticoagulants. Knowledge of anticoagulants was similar among genders, pharmacy locations, living situations, ability to self-manage medications, feelings of safety taking medications, and receipt of blood thinner education $(p>.05)$. Knowledge of anticoagulants differed among age groups, in years, and ethnicities $(p<.05)$. Patients 60 - 69 years old had an average actual knowledge score between 10 to 20 percent higher than any other age group $(p=.03)$. Computation of an average actual knowledge score for the 4049 years old age group did not occur due to only one patient represented. Caucasian Americans scored over $10 \%$ higher than African Americans ( $p=.04)$.

\section{Discussion}

This research sought to assess patients' perceived knowledge compared to their actual knowledge of their anticoagulants dispensed from an independent community pharmacy. 
Evaluating this comparison may stimulate similar discussion about other therapeutic classes. Study findings indicated patients' perceived and actual knowledge was largely similar across demographics. An important piece of the pharmacist interactions with patients after survey completions was that patients receiving refills on existing prescriptions received clarifying information about their anticoagulants that was unfamiliar to them previously. Incomplete knowledge of anticoagulant therapies commonly occurs among patients in various settings. ${ }^{6,9-10,12-14}$ Difficulties identifying notable side effects and drug interactions with anticoagulants are apparent. ${ }^{15-17,} 19$ Moreover, patients have demonstrated minimal understanding of their clinical conditions that lead to anticoagulant use. ${ }^{12}$

These reports might indicate the need for anticoagulant education that is more customizable to different literacy levels and available in settings conducive to informal dialogue between patients and healthcare practitioners.

A trend emerged in this study of declining actual anticoagulant knowledge extending from 60-69 years old to 80-89 years old with a more substantial decrease observed among patients in the 80-89 years old category. This finding is concerning as patients' use of anticoagulants can often increase with age. Cognitive impairment associated with the aging process coupled with patients taking other medications that possibly affect mental acuities probably factored in to this observation. Furthermore, percent of actual anticoagulant knowledge was disparate between ethnicities. Previous educational backgrounds in conjunction with variability in length of time having used anticoagulants along with twice the amount of study participation might have contributed to Caucasian Americans demonstrating greater than 10\% higher actual average anticoagulant knowledge than African Americans.

Over half of elderly patients in a different study defined as at least 60 years old with an average age of approximately 70 years old admitted to having difficulty remembering information about their anticoagulants. ${ }^{14}$ Less than half of elderly patients in this same study were cognizant of any adverse effects associated with their anticoagulants. ${ }^{14}$ The worsening of actual anticoagulant knowledge observed with advancing age in this study may be explained by similar recall deficits.

Patients of African descent in a separate investigation had less familiarity than White Europeans with the clinical conditions that precipitated their anticoagulant use. ${ }^{17}$ Diminished conceptions of physiologic abnormalities could translate to suboptimal understanding of corresponding anticoagulants. Caution is important when comparing individuals of African descent to African Americans along with White Europeans to Caucasian Americans.

However, African American patients in this study may have experienced similar challenges with understanding their anticoagulant-related disease states to the aforementioned patients of African descent. Therefore, this presumed parallel may account for African American patients displaying less actual anticoagulant knowledge than Caucasian American patients in this pharmacy setting.

Patients readily agreed to participate in this study seemingly as a way for them to learn more about their anticoagulants. Each anticoagulant contains unique therapeutic principles that are important to consider. Patients invariably were taking other medications apart from their anticoagulants positioning pharmacists to address any concerns relating to those therapies as well. The detailed approach to patient counseling at this independent pharmacy may not completely translate to pharmacy environments with greater prescription volumes and possibly less time to devote to individualized interactions.

There were some limitations to this study. Awareness of patient actual anticoagulant knowledge did not result from a validated assessment tool. Content presented in the survey assessments of perceived and actual anticoagulant knowledge did not completely align. Considering the cross-sectional nature of this research design, patients' may progress or regress in their understanding of anticoagulant medications going forward. Waning patient interest in the survey content or competing influences on their time as they navigated through to completion may have hampered reliability of the findings. An option was not available for patients to ask clarifying questions about the survey possibly leading to answers supplied that did not reflect total understanding of the content at hand.

Fewer than 50 participants comprising the study sample might limit the generalizability of the results generated here to populations that frequent other independent pharmacies.

The findings of this study are a prelude to a qualitative investigation. One of the authors of this study is preparing to facilitate individual interviews and focus group sessions with patients included and not included in this study to determine rationales for limited anticoagulant knowledge as a component of her post-graduate year one pharmacy residency experience. Affording patients the space to explain in their own words the disconnect that occurs with knowledge of their anticoagulant medication regimens is prone to place the results shown here into clearer context. Glimpses into anticoagulant understanding among independent pharmacy patients were not readily apparent prior to this study. Further investigations into anticoagulant and other drug awareness levels at different independent pharmacies could be insightful.

\section{Conclusion}

Sufficient knowledge of anticoagulants is typically lacking among patients utilizing these medications. Comfort should exist with details of anticoagulants prescribed for certain conditions. Patients in this study demonstrated a potential disconnect between perceived and actual knowledge of their anticoagulant therapies. The findings reported here support 
identifying customizable mechanisms for aiding in learning more about coagulated states, associated therapeutic management strategies, and recognition of indicators to alert healthcare professionals for rapid assistance.

Conflicts of Interest: We declare no conflicts of interest or financial interests that the authors or members of their immediate families have in any product or service discussed in the manuscript, including grants (pending or received), employment, gifts, stock holdings or options, honoraria, consultancies, expert testimony, patents and royalties.

\section{References}

1. Barber N, Parsons J, Clifford S, Darracott R, Horne R. Patients' problems with new medication for chronic conditions. Qual Saf Health Care. 2004;13(3)172-175.

2. Fuller R, Dudley N, Blacktop J. Avoidance hierarchies and preferences for anticoagulation - semi-qualitative analysis of older patients' views about stroke prevention and the use of warfarin. Age Ageing. 2004;33(6):608-611.

3. Dobesh PP, Fanikos J. Direct oral anticoagulants for the prevention of stroke in patients with nonvalvular atrial fibrillation: understanding differences and similarities. Drugs. 2015;75(14):1627-1644.

4. Fernandez MM, Scheele B, Hogue S, Kwong WJ. Review of challenges in optimizing oral anticoagulation therapy for stroke prevention in atrial fibrillation. Am J Cardiovasc Drugs. 2013;13(2):87102.

5. Pickett JD. Direct oral anticoagulants in patients with nonvalvular atrial fibrillation: update and periprocedural management. Am J Crit Care Nurses. 2019;39(20):54-67.

6. Metaxas C, Albert V, Habegger S, Messerli M, Hersberger KE, Anet I. Patient knowledge about oral anticoagulation therapy assessed during an intermediate medication review in Swiss community pharmacies. Pharmacy (Basel). 2020;8(2):e54.

7. Choudhury A, Lip GY. How good is anticoagulation control in non-valvar atrial fibrillation? Observations on the elderly, ethnicity, patient perceptions, and understanding of AF thromboprophylaxis. Heart. 2005;91(4):425-426.

8. Tseng AS, Schleifer JW, Shen WK, McBane R, Mankad $\mathrm{S}$, Esser H, Vucicevic D, Shamoun FE. Real-world incidence of efficacy and safety outcomes in patients on direct oral anticoagulants with left ventricular systolic dysfunction at a tertiary referral center. Clin Cardiol. 2017;40(12):1328-1332.

9. Ching TB, Sulaiman SA, Suleiman AK, Gillani SW, Abubakar U. Knowledge of warfarin therapy among patients attending warfarin clinic as a public hospital in northern part of Malaysian Peninsular. Arch Pharm Pract. 2016;7(1):14-17.
10. Kaufman BG, Kim S, Pieper K, Allen LA, Gersh BJ, Naccarelli GV, et al. Disease understanding in patients newly diagnosed with atrial fibrillation. Heart. 2018;104(6):494-501.

11. Lane DA, Meyerhoff J, Rohner U, Lip GY. Atrial fibrillation patient preferences for oral anticoagulation and stroke knowledge: results of a conjoint analysis. Clin Cardiol. 2018;41(6):855-861.

12. Reading SR, Go AS, Fang MC, Singer DE, Liu IA, Black $\mathrm{MH}$, Udaltsova $\mathrm{N}$, Reynolds K. Health literacy and awareness of atrial fibrillation. J Am Heart Assoc. 2017;6(4):e005128.

13. Rocha HT, Rabelo ER, Aliti G, Nogueira de Souza E. Knowledge of patients with mechanical valve prostheses concerning chronic oral anticoagulant therapy. Rev Latinoam Enferma. 2010; 18(4):696-702.

14. Koushal D. A survey study to assess the patients' knowledge about anticoagulation therapy. Int J Nurs Educ. 2018;10(3):144-147.

15. Joshua JK, Kakkar N. Lacunae in patient knowledge about oral anticoagulant treatment: results of a questionnaire survey. Indian J Hematol Blood Transfus. 2015;31(2):275-280.

16. Masnoon N, Sorich $\mathrm{W}$, Alderman CP. A study of consumer retention of key information provided by clinical pharmacists during anticoagulant counseling. $J$ Pharm Pract Res. 2016;46(3):227-244.

17. Nadar S, Begum N, Kaur B, Sandhu S, Lip GY. Patients' understanding of anticoagulant therapy in a multiethnic population. J R Soc Med. 2003;96(4):175179.

18. Zulkifly H, Cheli P, Lutchman I, Bai Y, Lip GYH, Lane DA. Anticoagulation control in different ethnic groups receiving vitamin $\mathrm{K}$ antagonist therapy for stroke prevention in atrial fibrillation. Thromb Res. 2020;192(1):12-20.

19. Saqib A, Atif M, Ikram R, Riaz F, Abubakar M, Scahill S. Factors affecting patients' knowledge about dispensed medicines: a qualitative study of healthcare professionals and patients in Pakistan. PLoS One. 2018;13(6):e0197482.

20. Yildiz, E, Dayapoglu N. The satisfaction levels of patients using anticoagulants. Int J Caring Sciences. 2017;10(1):568-574.

21. Bellamy L, Rosencher N, Eriksson BI. Access to a new oral anticoagulant treatment prescription: dabigatran etexilate. Patient Prefer Adherence. 2009;3(1):173177.

22. Mourad AP, Aslani P, D'Souza M, Brieger D. The acceptability of a direct oral anticoagulant monitoring regimen among patients with atrial fibrillation: a pilot study. Int J Clin Pharm. 2019;41(3):682-686. 
23. Cruess DG, Localio AR, Platt AB, Brensinger CM, Christie JD, Gross R, et al. Patient attitudinal and behavioral factors associated with warfarin nonadherence at outpatient anticoagulation clinics. Int J Behav Med. 2010;17(1):33-42.

24. Metlay JP, Hennessy S, Localio AR, Han X, Yang W, Cohen $A$, et al. Patient reported receipt of medication instructions for warfarin is associated with reduced risk of serious bleeding events. J Gen Intern Med. 2008;23(10):1589-1594. 
Table 1. Demographic distribution of patients among both independent pharmacy locations $(N=45)$

$\begin{array}{ll} & \frac{\text { Demographics }}{\text { Male }} \\ \text { Gender } \quad \text { Female }\end{array}$

Gender Female

$40-49$

50-59

$60-69$

Age group $\quad 70-79$

(years) $\quad 80-89$

Black / AA

Ethnicity White / CA

\section{Lugoff, SC (\%)}

16 (69.6)

$7(30.4)$

$1(4.3)$

$4(17.4)$

$4(17.4)$

$8(34.8)$

$5(26.1)$

$7(30.4)$

$16(69.6)$

5 (21.7)

$18(78.3)$

$0(0)$
Camden, SC (\%)

9 (40.9)

13 (59.1)

$0(0)$

5 (22.7)

7 (31.8)

8 (36.4)

$3(9.2)$

8 (36.4)

14(63.6)

7 (31.8)

$15(68.2$

$0(0)$

20(90.9)

$2(9.1)$

1 (4.3)

21 (91.3)

2 (8.7)
$18(81.8)$

2 (18.2)
Total (\%)

25 (55.5)

20 (44.5)

1 (2)

9 (20)

11 (24)

16 (36)

8 (18)

15 (33)

30 (66.7)

12 (26.7)

33 (73.3)

$0(0)$

Perceptions of anticoagulant safety

No

17 (73.9)

$17(77.3)$

$5(22.7)$

34 (75.6)

$6(26.1)$

$11(24.4)$

$39(86.7)$

$6(13.3)$

$42(93.3)$

3 (6.7) 
Table 2. Perceived anticoagulant knowledge of patients at both independent pharmacy locations $(N=45)$

$\begin{array}{ll} & \frac{\text { Demographics }}{\text { Male }} \\ \text { Gender } \quad \text { Female }\end{array}$

Gender Female

$\begin{array}{cll} & 40-49 & 1(92) \\ & 50-59 & 9(72) \\ \text { Age } & 60-69 & 11(75) \\ \text { group } & 70-79 & 16(77) \\ \text { (years) } & 80-89 & 8(82)\end{array}$

Ethnicity

$\begin{array}{ll}\text { Black / AA } & 15(73) \\ \text { White / CA } & 30(79)\end{array}$

Camden, SC

$22(75)$

Lugoff, SC

23 (78)

Location

$\begin{array}{llll} & \text { Alone } & 12(75) & .39 \\ & \text { With family } & 33(77) & (-4.5,5.2) \\ \text { Living situation } & \text { Institution/Facility } & 0(---)^{*}\end{array}$

\footnotetext{
${ }^{\ddagger}$ Anticoagulant management Yes

No

42 (77)

$3(71)$

Perceptions of anticoagulant safety Yes

No

34 (77)

11 (73)

Anticoagulant Yes

39 (75) education

No

.48

.65

$(-16.7,10.5)$

$(-1.8,19.1)$

$\mathrm{AA}=$ African American; $\mathrm{CA}=$ Caucasian American; $\mathrm{SC}=$ South Carolina

$\mathrm{Cl}=$ Confidence Interval; Score = Average rating on survey items.

${ }^{\ddagger}$ Participants attested to independently managing their anticoagulants.

${ }^{*}$ Not computed due to an absence of sample representation.
}

$95 \% \mathrm{Cl}$

$(-16.8,1.3)$

.09

$(-0.2,0.7)$

.26

$(-2.9,16.3)$

$(-12.7,5.7)$

$(-24.9,12)$ 
Table 3. Average actual anticoagulant knowledge of patients at both independent pharmacy locations $(\mathrm{N}=45)$

\begin{tabular}{|c|c|c|c|c|}
\hline & Demographics & $\mathbf{N}(\%$ Correct) & p-value & $\underline{95 \% \mathrm{Cl}}$ \\
\hline & Male & $25(74)$ & .26 & $(-16.5,4.5$ \\
\hline Gender & Female & $20(68)$ & & \\
\hline & $40-49$ & $1(100)^{ \pm}$ & † & $(-1.0,-0.1$ \\
\hline & 50-59 & $9(70)$ & בכ. & \\
\hline Age & $60-69$ & $11(81)$ & & \\
\hline group & 70-79 & $16(70)$ & & \\
\hline (years) & $80-89$ & $8(59)$ & & \\
\hline & Black / AA & $15(64)$ & $.04^{+}$ & $(0.3,21.7)$ \\
\hline & White / CA & $30(75)$ & & \\
\hline
\end{tabular}

Ethnicity

$\begin{array}{llll}\text { Camden, SC } & 22(71) & .44 & (-11.4,9.7) \\ \text { Lugoff, SC } & 23(72) & & \end{array}$

Location

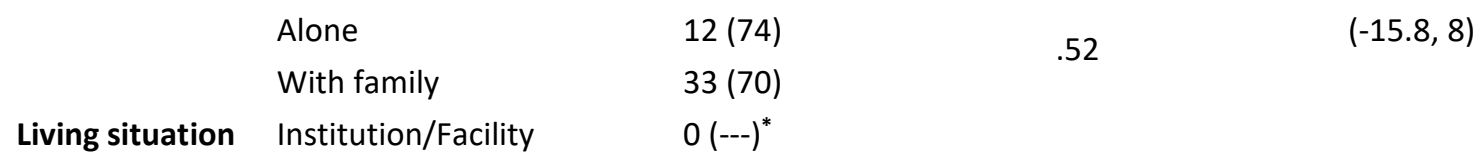

\begin{tabular}{|c|c|c|c|c|}
\hline \multirow{2}{*}{$\begin{array}{l}{ }^{\ddagger} \text { Anticoagulant } \\
\text { management }\end{array}$} & Yes & $42(72)$ & & \\
\hline & No & $3(63)$ & & \\
\hline \multirow{3}{*}{$\begin{array}{c}\text { Perceptions of } \\
\text { anticoagulant } \\
\text { safety }\end{array}$} & Yes & $34(71)$ & \multirow{2}{*}{.96} & \multirow[t]{2}{*}{$(-15.1,15.9)$} \\
\hline & No & $11(70)$ & & \\
\hline & & & & $(-1.6,22.2)$ \\
\hline \multirow{2}{*}{$\begin{array}{c}\text { Anticoagulant } \\
\text { education }\end{array}$} & Yes & 39 (69) & \multirow[t]{2}{*}{.09} & \\
\hline & No & $6(79)$ & & \\
\hline
\end{tabular}

${ }^{+} \mathrm{p}<.05$ considered statistically significant; $\mathrm{AA}=$ African American; $\mathrm{CA}=$ Caucasian American;

$\mathrm{SC}=$ South Carolina $\mathrm{Cl}=$ Confidence Interval; \% Correct = Average percent correct on survey items.

${ }^{\ddagger}$ Participants attested to independently managing their anticoagulants.

${ }^{*}$ Not computed due to an absence of sample representation. ${ }^{ \pm}$Actual as opposed to average percentage. 
Appendix A: Survey distributed to patients at both independent pharmacy locations

Section 1: Perceived Anticoagulant Understanding Assessment

Directions: On a scale of 1 to 5, 1 means not at all confident and 5 means very confident:

1. How confident are you that you know the reason you are taking this medicine?

$$
1
$$

3

4

2. How confident are you that you understand how to safely use your blood thinning medicine?

$\begin{array}{lllll}1 & 2 & 3 & 4 & 5\end{array}$

3. How confident do you feel that you understand the drug interaction potential of your blood thinning medicine?

$$
\begin{array}{lllll}
2 & 3 & 4 & 5
\end{array}
$$

4. How confident do you feel in your understanding of the side effects of your blood thinning medicine?

$$
\begin{array}{lllll}
1 & 2 & 3 & 4 & 5
\end{array}
$$

5. How confident are you that you would know what to do if you missed a dose of your blood thinning medicine?

1

2

3

4

5

Section 2: Actual Anticoagulant Understanding Assessment

(Note: bolded print among answer choices indicates correct response)

1. Which of the following would be a reason to visit an emergency room?

a. Scratching your arm on a tree branch while working in the yard.

b. Seeing tiny streaks of blood in the sink after brushing your teeth.

c. Noticing a large amount of bright red blood in the last $\mathbf{2}$ bowel movements.

d. Waking up with bad dreams and a dry mouth.

2. Which of the following procedures would you need to talk to you primary care doctor about your blood thinner before having?
a. Having a tooth pulled
b. Having a spinal injection
c. Having a surgery
d. All of the above

3. Why would your doctor decide to decrease the dose of your blood thinner?
a. Because I am at risk for bleeding
b. Because I was diagnosed with schizophrenia
c. Because my blood sugar is abnormal
d. None of the above

4. Which of the following over the counter medicines would you need to avoid taking with you blood thinner?
a. Multivitamin
b. Ibuprofen
c. Tylenol
d. Mucinex

5. If you were having mild pain in your back and needed to take an over the counter pain reliever, which would you choose?
a. Claritin
b. Ibuprofen
c. Tums
d. Tylenol 
6. If you missed a dose of your blood thinning medicine and it was time for your next dose what would you do?

a. Take two doses to catch up from the missed dose

b. Skip the missed dose and take the next scheduled dose

c. Wait to restart the medication tomorrow

d. Stop taking the medication

7. What could happen I forget to take my blood thinner?

a. I may have a seizure

b. I could form a blood clot

c. I may get a urinary tract infection

d. Nothing, it is not necessary for me to take this medication

8. Which side effect would require you to call your doctor immediately regarding your blood thinner?
a. Dizziness or passing out
b. Upset stomach
c. Headache
d. Runny nose

9. As a result of taking your blood thinning medicine, which of the following would you expect to notice?
a. Bruising
b. Urination
c. Sore throat
d. None of these

10. If you felt cold during the winter and thought your blood thinning medicine might be the cause, which would you do?
a. Stop taking the blood thinner while it is cold outside
b. Continue taking your blood thinner as usual
c. Take it when your blood pressure is high
d. Take an aspirin in place of the blood thinner 